\title{
Correction to: Abdominal fellowship-trained versus generalist radiologist accuracy when interpreting MR and CT for the diagnosis of appendicitis
}

\author{
Rebecca L. Bracken ${ }^{1}$ • John B. Harringa ${ }^{1}$ - B. Keegan Markhardt ${ }^{2,3} \cdot$ Newrhee Kim $^{2,3}$ - John K. Park ${ }^{2,3}$. \\ Douglas R. Kitchin ${ }^{2,4}$. Jessica B. Robbins ${ }^{2}$. Timothy J. Ziemlewicz ${ }^{2}$. Jen Birstler ${ }^{5}$ Michael J. Ryan ${ }^{1} \cdot$ Ly Hoang $^{1}$. \\ Perry J. Pickhardt ${ }^{2} \cdot$ Scott B. Reeder ${ }^{1,2} \cdot$ Michael D. Repplinger $^{1,2}$
}

Published online: 26 August 2021

(c) European Society of Radiology 2021

\section{Correction to: European Radiology}

https://doi.org/10.1007/s00330-021-08163-7

The original version of this article, published on 16 July 2021, unfortunately contained two mistakes. The following corrections have therefore been made in the original: The spelling of Newrhee Kim's name was incorrect and the middle name in Perry J. Pickhardt's name was missing. The corrected author list is given above.

The original article has been corrected.

Publisher's note Springer Nature remains neutral with regard to jurisdictional claims in published maps and institutional affiliations.

The original article can be found online at https://doi.org/10.1007/ s00330-021-08163-7.

Michael D. Repplinger

mdrepplinger@wisc.edu

1 BerbeeWalsh Department of Emergency Medicine,

University of Wisconsin-Madison, Madison, WI, USA

2 Department of Radiology, University of Wisconsin-Madison, Madison, WI, USA

3 Department of Radiology, UnityPoint Health Meriter, Madison, WI, USA

4 Madison Radiologists, Madison, WI, USA

5 Department of Biostatistics \& Medical Informatics, University of Wisconsin-Madison, Madison, WI, USA 